\title{
Emulating Full Load Testing of Air-cooled Nanocrystalline IHT at Zero Power
}

\author{
Arun Kumar Paul, Senior Member IEEE
}

\begin{abstract}
High-power high-frequency air-cooled induction heating transformers, mostly used as current multiplier and isolation purposes, are custom designed. For their reliable performance and long life, the thermal evaluation at rated load is necessary. Creating an equivalent load as test facility for reliability testing of such type of transformer is difficult. Characteristics of such loads drastically change after Curie temperature. Moreover, prolonged heating could increase the nearby ambient temperature and, more importantly, the traditional heat run test wastes large amount of energy. Whenever the coil is energized, windings of transformer draw respective rated current; even at no load condition the copper loss is at rated value. While both windings drawing rated current at desired frequency, using the concept of localized eddy current loss as well as excess eddy current loss, this article proposes a novel method to inject requisite core loss to the magnetic circuit to emulate the characteristics of full load condition but the power drawn from the transformer would be zero. The proposed idea would be validated where only $200 \mathrm{~W}$ resonant inverter would be used to inject power loss equivalent to full-load condition of $30 \mathrm{~kW}$ transformer to emulate the heat run test.
\end{abstract}

Keywords- Excess eddy current loss, induction heating transformer (IHT), magnetic loss, series resonant inverter (SRI), zero-voltage zero-current switching (ZVZCS).

\section{INTRODUCTION}

$\mathrm{H}$ IGH frequency high power transformers [1]-[10] for use in various power electronics converters are not commercially available. They are used not only for voltage/current multiplication or isolation purposes, their characteristics, often, make significant influence in converter design [11], [12]. In induction heating $(\mathrm{IH})$, nature of load decides the characteristics of power controller [13]. Therefore, transformers used therein are custom designed and often manufactured inhouse. It becomes important, at least through type testing, to verify their reliability performance. There are applications e.g., post weld heat treatment of welded pipes [14] where for soaking the pipe at $750{ }^{\circ} \mathrm{C}$ the induction heating transformer (IHT) needs to deliver full capacity continuously for more than ten hours.

Optimal design of transformer involves maximal use of core and copper [4] provided both are within their respective thermal limits [8], [9]; the power density should be large. Power loss in both core and copper should be minimized and heat conduction features of core and winding need to be improved. With the introduction of ribbon-structured low loss thermally conductive

The author is with Messieurs Electronics Devices World Wide Pvt. Ltd, 22 Mistry Industrial Estate, MIDC, Andheri, Mumbai 400093, India. (email: arunp26@iitbombay.org). nanocrystalline soft magnetic materials [5] and availability of thinner gauge litz-wire conductors the power loss in core and copper has been reduced significantly [3]. High operating flux density of nanocrystalline cores help reduce the volume of copper needed in windings, the copper loss is accordingly reduced. Still, the heat removal features from core and windings are not uniform [8]. Moreover, multiple heat sources exist in the transformer. Generation of hot spots could be prevalent.

In high power IH applications the coil current is large, transformer is needed for necessary current multiplication (see Fig. 1). Traditionally, they are conduction-cooled. Due to availability of both ribbon type nanocrystalline cores with large window area and wide range litz wire conductors, several application areas (e.g., where the density of transferred power $\left(\mathrm{W} / \mathrm{cm}^{2}\right)$ on job surface is moderate) are now being covered by air-cooled controllers [14] where IHT is an integral part. High permeability and low leakage flux make nanocrystalline cores ideally suitable for IHT used by ZVZCS SRI [3], [7].

Due to windings placed around the core, the heat removal features of each segment of core and copper could be different. Proper thermal evaluation is necessary. Organizing a facility for application-sensitive full load testing of IH system is difficult. It could increase the temperature of the ambience. Moreover, the load would mostly disappear once the job attains the Curie temperature. Such type of thermal evaluation would waste large amount of energy. It is known that, if power loss and thermal conduction characteristics of IHT are modeled properly then the finite element method (FEM) [8] could be used to predict the temperature profile in core and copper accurately. Still, to validate results of FEM similar complex test facility is desired. In this article, a novel practical method to emulate full-load testing of IHT is proposed. Here, proper thermal evaluation of IHT is performed by ensuring power loss in both core and copper to respective rated value, but the power drawn from the IHT would be zero. The structure of the article is as follows: Section II briefly discusses the IHT and approach for its optimal design. Section III discusses the loss characteristics of the transformer. Section IV details the utility of ZVZCS SRI for evaluation of functioning of IHT. It also elaborates the proposed idea of how quantum of power could be injected into the core of IHT even when power drawn from it is negligible. Finally, Section V further strengthened the novel idea of practically emulating the full load testing of IHT without extracting any power from it, but secondary current at rated value is mandatory requirement. The proposed idea is more suitable for cores with good thermal conductivity. 


\section{Induction Heating Transformer: Design Issues}

SRI topology (see Fig. 1) is most popularly used to feed the load using induction heating principle. To facilitate transfer of large power in non-contact mode the coil current is kept large. IHT is used for necessary current multiplication. The controller uses two control loops - phase locked loop (PLL) is used to track the resonant frequency of tank circuit and chopper is used to control the power delivered to metallic object. The design of IHT involves on deciding the number of primary and secondary turns, selection of core material, core geometry, litz wires of proper size, winding patterns, thermal management, etc. Ideally, for its optimal design, the power loss in core and copper should be minimized and layout of windings should be able to remove the heat loss efficiently so as to keep their temperature rise with in the respective limits. For reduction of highfrequency copper loss, single-layered windings are preferred because the impact of proximity effects becomes negligible. Single-layered windings are feasible when the number of turns at primary $\left(n_{\mathrm{p}}\right)$ and at secondary $\left(n_{\mathrm{s}}\right)$ should be at respective minimum permissible value, it laterally improves thermal conduction characteristics. Large value of turns ratio $n$ improves the performance of the controller because the primary side current is also reduced. The number of primary turns $n_{\mathrm{p}}$ is decided by input conditions of IHT, as,

$n_{p}=\frac{V_{\mathrm{pri}}(\mathrm{FL})}{4 B_{\mathrm{m}} A_{\mathrm{core}} f_{s}}$

$V_{\text {pri }}(\mathrm{FL})$ is the square wave primary input voltage at full load, $B_{\mathrm{m}}$ is peak flux density in core of effective area $A_{\text {core }}$ and $f_{\mathrm{s}}$ is switching frequency of inverter.

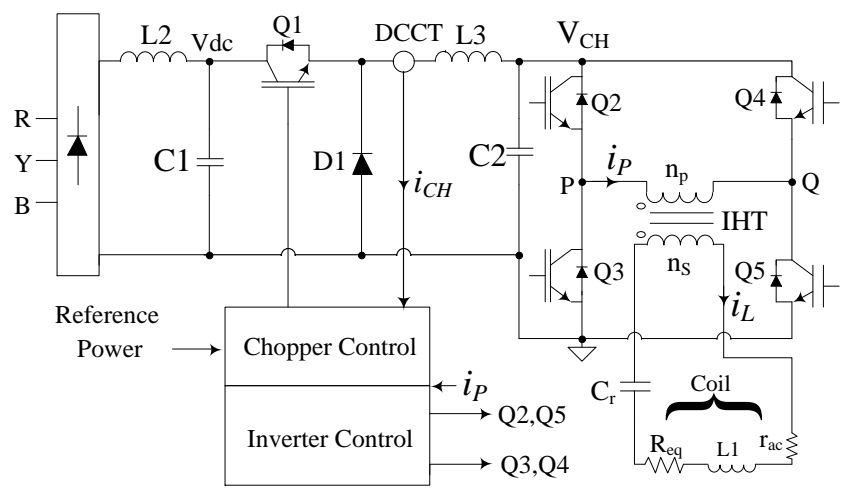

Fig. 1: Series resonant power controller for induction heating

\section{A. Optimal Turns Ratio for Controller Optimization}

The input $V_{\text {pri }}$ to IHT to feed resonant tank circuit is square wave, but the current $i_{\mathrm{p}}$ at primary or $i_{\mathrm{L}}$ at secondary would be pure sinusoidal because at any harmonic frequency other than the fundamental the tank circuit impedance is large. Turns ratio $n$ needed to feed rated power to load is expressed as,

$n=\frac{n_{p}}{n_{S}}=1.1 \frac{\mathrm{V}_{\mathrm{pri}}(\mathrm{FL})}{Z_{\mathrm{eq}} i_{L}}$

$Z_{\text {eq }}$ is the tank circuit impedance, whose expression is,

$Z_{\mathrm{eq}}=\left(R_{\mathrm{eq}}+\mathrm{esr}+r_{\mathrm{eq}}\right)+j\left(2 \pi f_{s} L_{1}-\frac{1}{2 \pi f_{s} C_{r}}\right)$
$R_{\text {eq }}$ is effective load resistance, $r_{\text {ac }}$ is ac coil resistance, esr is resistance of tank capacitor $\mathrm{Cr}$ and $f_{\mathrm{s}}$ is switching frequency of inverter. Under ZVZCS condition, $Z_{\mathrm{eq}}$ is minimum at $\left(r_{a c}+\right.$ esr $\left.+R_{e q}\right)$ where the value of $n$ could be maximum at $n_{\max }$, at,

$n_{\max }=\frac{n_{p}}{n_{s}(\min )}=1.1 \frac{V_{p r i}(\max )}{\left(r_{\mathrm{ac}}+e s r+R_{\mathrm{eq}}\right) i_{L}}$

For constant coil current, the turns ratio at $n_{\max }$ minimizes the current in primary winding and the length of secondary winding is reduced, they together help minimize copper loss $P_{\mathrm{cu}}$.

Under ZVZCS condition, $V_{\text {pri }}$ may be approximated as,

$V_{\mathrm{pri}}=\frac{2 \sqrt{2}}{\pi} n\left(\frac{R_{\mathrm{pri}}}{n^{2}}+R_{\mathrm{sec}}+\mathrm{esr}+r_{\mathrm{ac}}+R_{\mathrm{eq}}\right) i_{\mathrm{L}}$

$R_{\text {pri }}$ and $\mathrm{R}_{\mathrm{sec}}$ respectively are primary and secondary resistance of IHT.

There are more reasons to choose the ZVZCS topology. It minimizes the power loss in inverter by virtually eliminating switching losses in IGBTs Q2-Q5. It also optimizes the conduction loss because there is no circulating current in inverter. RMS current in other power components is also at respective minimum value. For square wave $V_{\text {pri }}$ and sinusoidal input current $i_{\mathrm{p}}$ the input power $P_{\text {in }}$ could be expressed as,

$P_{\text {in }}=0.9 V_{\text {pri }} i_{\mathrm{p}}$

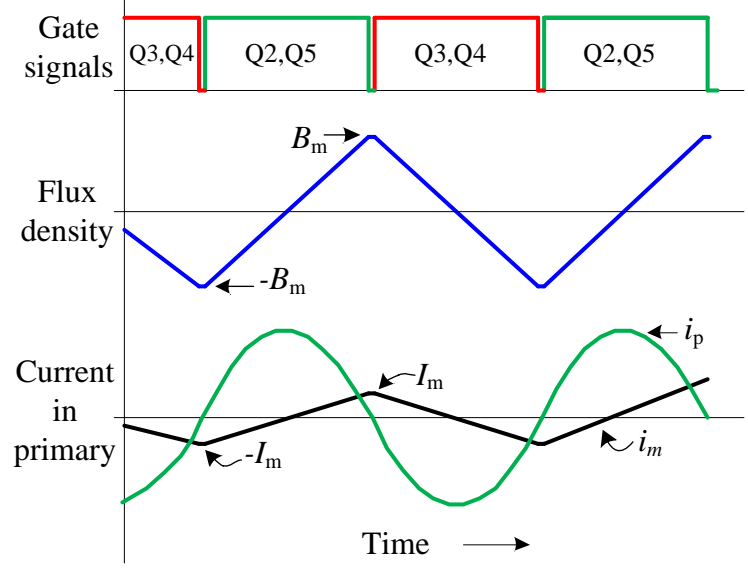

Fig. 2: Magnetizing current $i_{\mathrm{m}}$ could make impact on ZCS operation

\section{B. Optimal ZVZCS Functioning}

In the controller, PLL and chopper controller together ensure ZVS and ZCS operation of inverter. However, sensing of phase angle between fundamental of $V_{\text {pri }}$ and $i_{\mathrm{p}}$ is important. With balanced gate drives for square wave $V_{\text {pri }}$ the primary current $i_{\mathrm{p}}$ consists of two components (see Fig. 2) - the triangular wave magnetizing current $i_{\mathrm{m}}$ plus the reflected sinusoidal coil current $i_{\mathrm{L}}$. In ZVZCS condition, $i_{\mathrm{p}}$ is in phase with $V_{\text {pri. }}$. The peak of $i_{\mathrm{m}}$ i.e., $I_{\mathrm{m}}$ occurs when a pair of devices of inverter switching off. If its value is large then the value of $f_{\mathrm{s}}$ would be less than desired $f_{\mathrm{r}}$ and current $i_{\mathrm{L}}$ would be lagging the input voltage $V_{\text {pri. }}$. For ZCS operation of inverter switches, the value of $I_{\mathrm{m}}$ should be small. It is expressed as,

$I_{m}=\frac{l_{\mathrm{mag} B_{m}}}{\mu_{0} \mu_{r} n_{p}}$ 
Where $l_{\text {mag }}$ is mean length of core having relative permeability $\mu_{\mathrm{r}}$. For minimum value of $I_{\mathrm{m}}$, the core should have large value of $\mu_{\mathrm{r}}$ where high permeability toroidal cores are suitable.

\section{$C$. Role of Transformer on Power Delivery}

The power $P_{\text {Out }}$ transferred to load at coil current $i_{\mathrm{L}}$ is,

$P_{\text {OUT }}=i_{L}^{2} R_{\text {eq }}$

When the coil is loaded, due to secondary effect of load the inductance L1 drifts to, say, $L_{\mathrm{eq}}$. Expressions of $R_{\mathrm{eq}}$ and $L_{\mathrm{eq}}$ are,

$R_{\mathrm{eq}}=\frac{\omega_{S}^{2} M^{2} R_{\mathrm{wp}}}{R_{\mathrm{wp}}^{2}+\omega_{s}^{2} L_{\mathrm{wp}}^{2}}$ and $L_{e q}=L_{1}-\frac{\omega_{S}^{2} M^{2} L_{\mathrm{wp}}}{R_{\mathrm{wp}}^{2}+\omega_{s}^{2} L_{\mathrm{wp}}^{2}}$

$L_{\mathrm{wp}}$ is inductance and $R_{\mathrm{wp}}$ is resistance of workpiece, $M$ is mutual inductance between coil and workpiece and $\omega_{s}=2 \pi f_{s}$.

When L1 loaded, resonant frequency $f_{\mathrm{r}}$ is drifted to $f_{\text {eq }}$, like,

$f_{\text {eq }}=\frac{1}{2 \pi \sqrt{\left(L_{\text {leak }}+L_{\text {eq }}\right) C_{r}}} \approx f_{S}$

$L_{\text {leak }}$ is leakage inductance of IHT. For effective power transfer the load applied on coil should make direct impact on $f_{\mathrm{s}}$ where large value of $L_{\text {leak }}$ could be a hindrance. Here, as well, high permeability toroidal shaped cores are more suitable.

TABLE I

DETAILED PARAMETERS OF TANK CIRCUIT AND $30 \mathrm{~kW}$ IHT

\begin{tabular}{cc}
\hline \hline Parameter & Value \\
\hline Primary and secondary turns & 9 and 3 \\
Core material of IHT & Nanocrystalline \\
Steinmetz parameters $K_{\mathrm{s}}, \alpha, \beta[15]$ & $1.015,1.4364,1.638$ \\
Value of $\mu_{\mathrm{r}}$ at $B_{\mathrm{m}}=0.458 \mathrm{~T}$ & 29780 \\
Ribbon thickness, $\mu \mathrm{m}$ & 30 \\
Peak magnetizing current $I_{\mathrm{m}}, \mathrm{A}$ & 0.42 \\
Mean length of core $l_{\mathrm{mag}}, \mathrm{m}$ & 0.29 \\
Total core area, cm ${ }^{2}$ & 21.6 \\
Total core weight, $\mathrm{kg}$ & 4.64 \\
Power input to IHT @ 460V, $75 \mathrm{~A}, \mathrm{~W}$ & 31000 \\
Core loss $P_{\text {core }}$ 0.458 T, $12.9 \mathrm{kHz}, \mathrm{W}$ & 51.6 \\
Total copper loss $P_{\mathrm{cu}}, \mathrm{W}$ & 94.3 \\
Efficiency for sinusoidal excitation, $\%$ & 99.53 \\
Thermal conductivity of core, $\mathrm{W} / \mathrm{mK}$ & 10 \\
Capacitor Cr, $\mu \mathrm{F}$ and its esr, $\mathrm{m} \Omega$ & $6.5,0.3$ \\
Inductance of coil head $\mathrm{L} 1, \mu \mathrm{H}$ & 26.5 \\
Rated coil current, A & 225 \\
Current density in winding conductors, A/mm2 & 3.80 \\
Strand dia. of litz wires in both windings, mm & 0.2 \\
\hline \hline
\end{tabular}

\section{Testing of $30 \mathrm{~kW} \mathrm{IHT} \mathrm{at} \mathrm{Rated} \mathrm{Load}$}

It is clear that core material and its geometry play influential roles to optimize not only the IHT but also the controller. Here, nanocrystalline material [3], [5] is preferred because of its,

1. Small core loss density, $\mathrm{W} / \mathrm{kg}$

2. Large value of $\mu_{\mathrm{r}}$ at operating flux density $B_{\mathrm{m}}$ helps reduce the value of $I_{\mathrm{m}}$. Its large value could introduce an error in sensing of phase angle between $V_{\text {pri }}$ and $i_{\mathrm{L}}$.

3. Large value of saturation flux density $B_{\text {sat }}$

4. Large thermal conductivity could avoid creation of any hot spot in the core and, importantly,
5. Robust parametric behavior; parameters of cores such as $B_{\text {sat }}$ and $\mu_{\mathrm{r}}$, etc. are virtually insensitive to operating conditions of core and temperature.

To understand its functioning for feeding a series tank circuit, an air-cooled $30 \mathrm{~kW}$ medium frequency IH controller was built. The complete system is shown in Fig. 3. Details of the experimental set up along with parameters of IHT and tank circuit are listed in Table I. The resonant frequency $f_{\mathrm{r}}$ of the unloaded tank circuit was $12.1 \mathrm{kHz}$. To understand the loss characteristics of IHT the system was tested at full load. And, to determine the value of $\mu_{\mathrm{r}}$ of the core [15] accurately at the operating value of $B_{\mathrm{m}}$, the value of $I_{\mathrm{m}}$ was first determined by keeping the secondary open, PLL was disabled.

Fig. 4a shows the waveforms of the inverter at rated load of $30 \mathrm{~kW}$, the measured value of $V_{\text {pri }}$ was $460 \mathrm{~V}$ where the calculated value of $B_{\mathrm{m}}$ was at $0.458 \mathrm{~T}$. The efficiency of IHT was $99.53 \%$. Fig. $4 \mathrm{~b}$ shows the waveforms of $V_{\text {pri }}$ and the magnetizing current $i_{\mathrm{m}}$ because the secondary was kept open. At $B_{\mathrm{m}}$ of $0.458 \mathrm{~T}$, the value of $I_{\mathrm{m}}$ was $0.42 \mathrm{~A}$ and the corresponding value of $\mu_{\mathrm{r}}$ of core was 29780 .

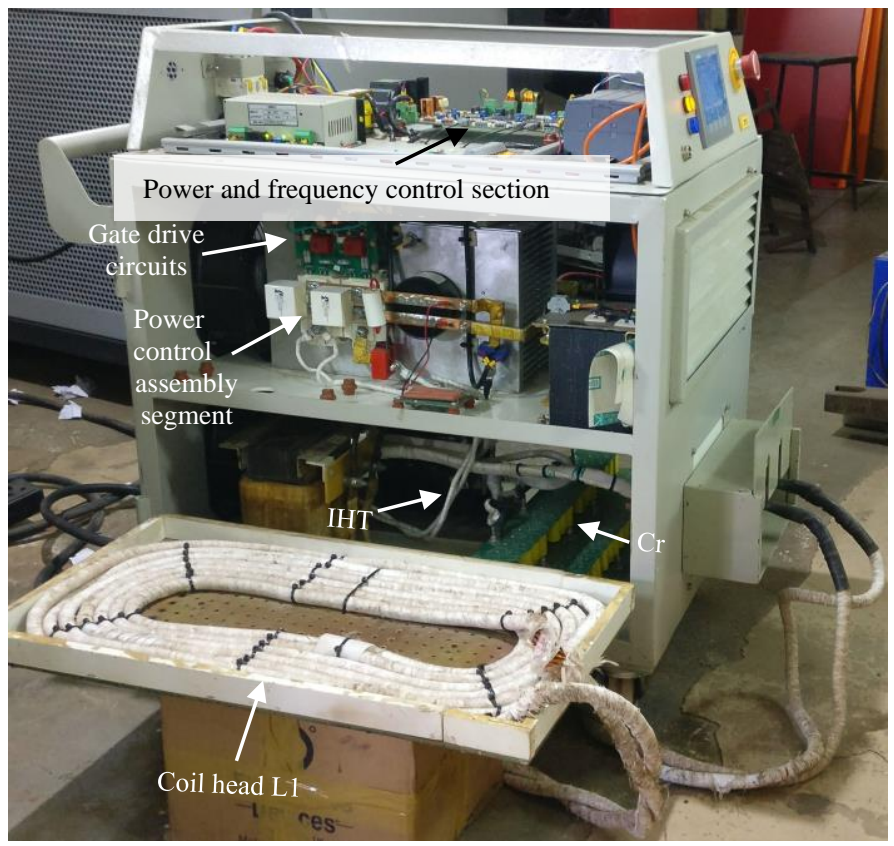

Fig. 3: Complete $30 \mathrm{~kW}$ air-cooled induction heating system, L1 not loaded

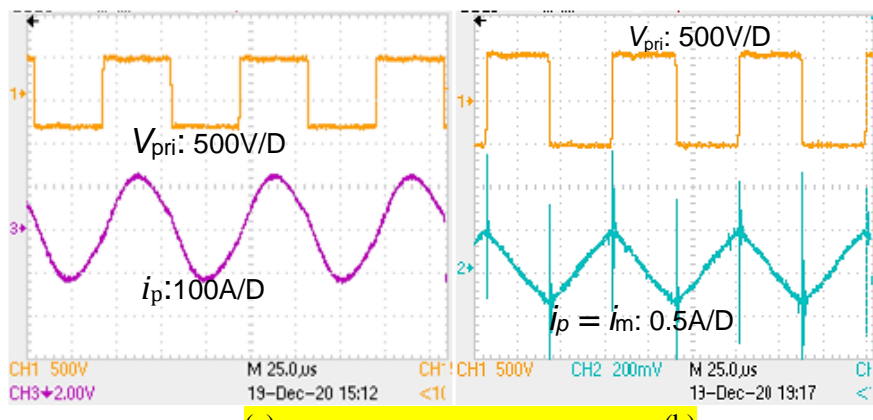

(a)

(b)

Fig. 4: a) Waveforms of $V_{\text {pri }}$ and $i_{\mathrm{p}}$ at input power of $31.0 \mathrm{~kW}$ at $12.9 \mathrm{kHz}$, and, b) the corresponding magnetizing current was obtained at $511 \mathrm{~V}, 14.35 \mathrm{kHz}$ with secondary kept open (PLL disabled), at $B_{\mathrm{m}}$ of $0.458 \mathrm{~T}$ the value of $I_{\mathrm{m}}$ of $0.42 \mathrm{~A}$. 


\section{LOSS CHARACTERISTICS OF IHT}

The total power loss in a transformer, consists of two parts the core loss $P_{\text {core }}$ and the copper loss $P_{\text {cu. Individual loss }}$ components are expressed as,

$$
\begin{aligned}
& P_{\text {core }}=W_{\text {core }} P_{\mathrm{c}}=W_{\text {core }} K_{S} f_{S}^{\alpha} B_{m}^{\beta} \\
& \text { and } P_{\text {cu }}=i_{L}^{2}\left(\frac{R_{\text {pri }}}{n^{2}}+R_{\text {sec }}\right)
\end{aligned}
$$

$P_{\mathrm{c}}$ is core loss density $(\mathrm{W} / \mathrm{kg}), W_{\text {core }}$ is core weight. $K_{\mathrm{S}}, \alpha$ and $\beta$ are Steinmetz parameters. $R_{\text {pri }}$ and $R_{\mathrm{sec}}$ respectively are $a c$ resistance of primary and secondary windings. Eqn. (10) is suitable for sinusoidal voltage, for other waveforms suitable multiplication factor is attached to it [2].

Ideally, power loss needs to be minimized. $P_{\text {core }}$ depends on several factors such as chosen soft magnetic material, magnitude, frequency and waveform pattern of primary voltage, etc. On the other hand, $P_{\text {cu }}$ depends on current density, strand size of litz wire, winding layout, current harmonics, proximity effects and copper temperature. If both windings laid in single layers, it would reduce the impact of high-frequency proximity effects on $P_{\text {cu }}$ [4], it also simplifies the thermal conduction features to improve removal of heat from windings.

While delivering rated power, the resulting power loss in IHT would increase the temperature of core and copper windings. The distribution of temperature in various segments of core and windings depends on respective heat conduction characteristics. For complex arrangement of core and windings, FEM [8] is often applied to determine the temperature distribution so as to locate the hot spot temperature. The approach is quite useful to optimize the design of IHT.

The approach to draw power from IHT is different from conventional transformers. Though, the functioning is same, IHT is regarded as current multiplier because the coil L1 is kept energized at rated current to facilitate power transfer to job. Both windings always draw respective rated current. The value of $B_{\mathrm{m}}$ in core depends on quantum of power delivered to metallic job. Here, $P_{\text {cu }}$ is fixed at rated value, but $P_{\text {core }}$ depends on quantum of power being transferred.

\section{A: Copper Loss Characteristics of IHT}

For series resonant tank-circuit the current waveform in both windings is pure sinusoidal. Secondly, for fundamental current, the impact of skin effect on copper loss is ignored because the skin depth $(=0.571 \mathrm{~mm})$ is much larger than the diameter $(=0.2$ $\mathrm{mm}$ ) of litz wire strands. If each winding is laid in single layer, for such conductors the impact of proximity effects on copper loss could as well be neglected [4].

In SRI fed IH system, even at no load, the value of $P_{\text {cu }}$ is at its rated value. For optimal energy efficiency, its value should be minimized first. Eqn. (11) could be re-written as,

$P_{\text {cu }}=k_{\text {pri }} \frac{i_{p}}{A_{p}} \rho l_{p} i_{p} n_{p}+k_{\text {sec }} \frac{i_{L}}{A_{s}} l_{p} \rho n_{s} i_{L}$

Where $k_{\text {pri }}$ and $k_{\text {sec }}$ respectively are ac resistance factor of primary and secondary conductors with $A_{\mathrm{p}}$ and $A_{\mathrm{s}}$ is respective copper area, $l_{\mathrm{p}}$ is mean length per turn and $\rho$ is resistivity of copper. With both windings laid in single layers and with the designed ratio of diameter of strands of round litz wire conductors to skin depth at 0.35 , the impact of skin and proximity effects on ac resistance value would be negligible [4], [6]; value of $k_{\text {pri }}$ and $k_{\mathrm{sec}}$ is considered at unity. Further, considering the same value of current density $J$ for both the windings, (12) may be simplified as:

$P_{\mathrm{cu}}=J \rho l_{p}\left(i_{p} n_{p}+n_{s} i_{L}\right)=2 J \rho l_{p} n_{s} i_{L}$

Cost factor needs optimal use of copper, if permitted by thermal considerations, the value of $J$ should be large. For minimal copper volume, both $n_{\mathrm{p}} l_{p}$ and $n_{\mathrm{s}} l_{\mathrm{p}}$ should be at respective minimum value. It helps windings have optimum conductor layout to reduce the impact of high-frequency proximity effects on $P_{\mathrm{cu}}$. It also helps improve the heat removal feature and the value of $L_{\text {leak }}$ of (9) is also reduced [10].

\section{B: Core Loss Characteristics of IHT}

With given value of $B_{\mathrm{m}}$ and $f_{\mathrm{s}}$, (10) is universally used to calculate core loss in soft magnetic materials where Steinmetz parameters are derived from the loss curves given in product datasheet. Considering uniform distribution of $B_{\mathrm{m}}$, ideally, $P_{\mathrm{c}}$ of (10) [16], [17] consists of hysteresis loss $P_{\mathrm{h}}$ and eddy current loss $P_{\text {el. }} P_{\mathrm{c}}$ of (10) should be matched by the formula,

$P_{\mathrm{c}}=P_{\mathrm{h}}+P_{\mathrm{el}}=k_{h} f_{s} B_{m}^{p}+k_{e} f_{s}^{2} B_{m}^{2}$

For a particular material, parameters $k_{\mathrm{h}}, k_{\mathrm{e}}$ and $p$ are considered as constant. In reality, there exists a large gap between the measured core loss density of (10) and the mathematically derived one in (14). Even for perfectly overlaid windings (see Fig. 5), the skin effect in ribbons of core makes significant influence on distribution of $B_{\mathrm{m}}$ [18], [19], particularly, in high permeability electrically conductive nanocrystalline cores. The said gap between (10) and (14) is more for cores of thicker ribbons and/or operating at higher frequency of excitation [18]. To match the actual core loss of (10), as a correction, a new component of magnetic loss i.e., the excess eddy current loss $P_{\text {ex }}$ [16], [17], [20, [21] has been introduced. It occurs due to non-uniform distribution of flux density in ribbons or core. The expression of $P_{\mathrm{ex}}$ is,

$P_{\mathrm{ex}}=k_{\mathrm{ex}} f_{s}^{1.5} B_{m}^{1.5}$

The parameter $k_{\mathrm{ex}}$ depends on ribbon thickness, skin depth, conductivity of material, etc. Uniformity in distribution of $B_{\mathrm{m}}$ could be affected radially through skin depth, it could as well be affected circumferentially if the coupling between windings is not proper [22]. The leakage inductance would contribute to localized concentration of flux density. Therefore, by reducing the coupling between the primary and secondary windings, power loss could be injected into the core.

\section{Emulating Full LoAd Power Loss Condition of IHT AT ZERO OUTPUT POWER}

The aim of reliability testing of power electronics product is to find that each component is within the safe temperature limit. It is of particular interest for customized component such as IHT because it has multiple heat sources with complex heat conduction features [9]. For reliable performance, accurate evaluation of temperature profile of core and two windings at rated load conditions is required. Though, FEM [8] could be used to predict temperature profile on core and windings, it requires accurate modeling of complex loss characteristics, 
distribution power loss in core and copper and, also modeling of dynamic heat conduction characteristics. Still, to validate the results of FEM the IHT would need to go through full load testing. The arrangement to conduct prolonged full load testing of high-power IH controller is elaborate because of following reasons:

1. The loading arrangement is application dependent, best suited at customer's site

2. Each equipment needs separate facility

3. Loads are functional till Curie temperature

4. For heat run test, it needs strong cooling arrangement that, as well, consumes extra energy, and,

5. There is large waste of energy

To conduct the reliability test of IHT, an alternate approach of injecting power equivalent to rated core loss value into the core is detailed here. It is simple because the quantum of power required to be handled is negligible.

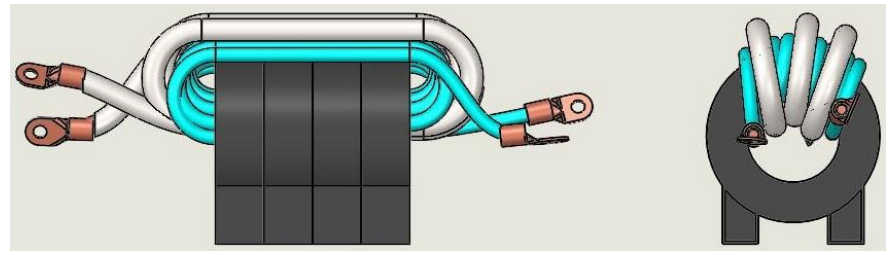

Fig. 5: Views of single-layer overlaid windings, such configuration has small value of leakage inductance and better distribution of $B_{\mathrm{m}}[22]$

\section{A. Insights of No-Load Testing of ZVZCS Driven IH System}

It is now clear that, in series tank-circuit, to facilitate energy transfer to load in non-contact mode, L1 is kept energized to its rated current of specified frequency. Irrespective of the loading condition the value of $P_{\mathrm{cu}}$ is at its rated value. It alone, however, cannot be used to validate the design of two windings. The core loss and its distribution make large influence on transfer of heat from windings. To assist the process, the design of IHT, therefore, should not only minimize $P_{\text {core }}$ but also ensure its uniform distribution [22]. In that sense, the no-load testing of SRI could indirectly be used to study the quality of design of IHT, its perfectness would be reflected on the value of resonant frequency $f_{\mathrm{r}}$. Its value should be maximum and corresponding value of $V_{\text {pri }}$ or $B_{\mathrm{m}}$ in (1) is minimum. Interestingly, the ZVZCS inverter is equipped to differentiate between different winding configurations and can decide what is best suited. For proper winding configuration i.e., when two single-layer windings are laid exactly one above the other (see Fig. 5) the value of noload primary voltage $V_{\text {pri }}(\mathrm{nl})$ would be minimum (so is $B_{\mathrm{m}}$ ) and the phase locked loop would result maximum value of $f_{\mathrm{s}} \approx f_{\mathrm{r}}$. It essentially means the value of $L_{\text {leak }}(9)$ is minimum as demonstrated in the expression below,

$f_{\mathrm{r}}=\frac{1}{2 \pi \sqrt{\left(L_{\text {leak }}+L_{1}\right) C_{r}}}$

Using (5), corresponding no-load power input $P_{\text {in }}(\mathrm{nl})$ to IHT is,

$P_{\text {in }}(\mathrm{nl})=0.9 V_{\text {pri }}(\mathrm{nl}) i_{p}$

For overlaid windings configuration (see Fig. 5), the waveforms of the actual system are shown in Fig. 6a. The measured value of $V_{\text {pri }}(\mathrm{nl})$ was approximately at $9.1 \mathrm{~V}$.
Corresponding calculated value of $B_{\mathrm{m}}$ was $9.6 \mathrm{mT}$ and that of $P_{\mathrm{c}}$ was approximately $18 \mathrm{~mW} / \mathrm{kg}$. Therefore, at no load, compared to large value of $P_{\mathrm{cu}}$ at $94.3 \mathrm{~W}$ (see Table I), for perfectly overlaid windings, $P_{\text {core }}$ could be neglected.

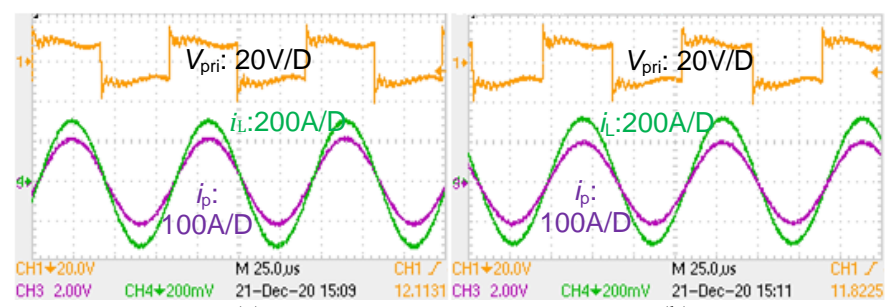

(a)

(b)

Fig. 6: Waveforms of $V_{\text {pri }}$ and $i_{\mathrm{p}}$ when the coil was not loaded, a) for perfectly overlaid windings where average value of $V_{\mathrm{CH}}$ was $12.3 \mathrm{~V}$ and $f_{\mathrm{r}}$ was 12.15 $\mathrm{kHz}$, and, b) when secondary was shifted by $30^{\circ}$ where average $V_{\mathrm{CH}}$ was 13.2 $\mathrm{V}$ and $f_{\mathrm{r}}$ was $11.9 \mathrm{kHz}$, but $P_{\text {core }}$ injected was $60.7 \mathrm{~W}$.
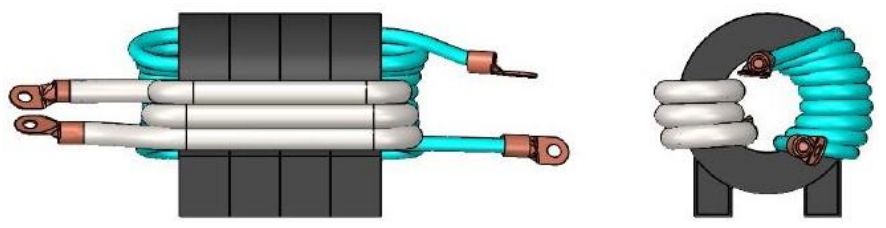

Fig. 7: Single layer primary and secondary windings not laid one above the other, have non-uniform distribution of $B_{\mathrm{m}}$ in the core [22]

\section{B. Injection of Rated Core Loss into the Core at No Load}

At full load condition (see Fig. 4a and Table I), the calculated value of $B_{\mathrm{m}}$ was $0.458 \mathrm{~T}$. Parametric details of nanocrystalline cores used here are listed in Table I [15]. Considering sinusoidal excitation, the corresponding value of $P_{\text {core }}$ was $51.6 \mathrm{~W}$. Can this or the quantum of power equivalent to square wave excitation $(>51.6 \mathrm{~W})$, with both windings drawing respective rated current, be injected into the core even when no power was exhausted through L1? The answer is yes. Here, a novel yet practical approach is followed to inject the requisite loss in core to find the temperature profile of core and copper.

Unlike ferrites, $P_{\mathrm{el}}$ and $P_{\mathrm{ex}}$ are dominant components of $P_{\text {core }}$ in transverse field annealed nanocrystalline cores, normally used in high power applications [18]. It was detailed in last subsection that for perfectly overlaid windings the leakage flux was minimum and $B_{\mathrm{m}}$ was uniformly distributed in core. The value of both $P_{\mathrm{el}}$ and $P_{\mathrm{ex}}$ was small [17]-[21]. What would happen if one winding is physically shifted from the other? It has been detailed in [22] that, by doing so, there is increase in leakage inductance by, say, $l_{1}$ and there is reduced uniformity in distribution of flux density in core. It would result in increase in core loss, more particularly, in $P_{\text {ex }}$. ZVZCS SRI is equipped to track both the changes accurately. The excess leakage would result in decrease in $f_{\mathrm{r}}$ to say $f_{\mathrm{r} 1}$, like,

$f_{\mathrm{r} 1}=\frac{1}{2 \pi \sqrt{\left(L_{\text {leak }}+l_{1}+L_{1}\right) C_{r}}}$

Where $l_{1}$ is the extra leakage inductance of IHT under test, contributed by shifting one winding relative to the other. Such shifting, through non-uniform distribution of $B_{\mathrm{m}}$, would incur extra loss in core, it would be reflected in an increase in primary 
voltage from $V_{\text {pri }}(\mathrm{nl})$ of (17) to pseudo-load condition, say, $V_{\text {pri }}(\mathrm{pl})$. They are related as,

$$
\begin{aligned}
& 0.9 V_{p r i}(\mathrm{pl}) i_{p}-0.9 V_{p r i}(\mathrm{nl}) i_{p}=P_{e x}+\Delta P_{e l} \\
& \text { Or, } V_{p r i}(\mathrm{pl})-V_{p r i}(\mathrm{nl})=\Delta V_{p r i}(\mathrm{pl})=\frac{\left(P_{e x}+\Delta P_{e l}\right)}{0.9 i_{p}}
\end{aligned}
$$

Table II details the situation, practically explored, when the angle between secondary and primary is gradually increased from zero. It appears that there is loss in uniformity in $B_{\mathrm{m}}$, it is gauged by an increase in leakage inductance $l_{1}$, and the worstcase situation [22] occurs when the angle is $180^{\circ}$ (see Fig. 7).

TABLE II

IMPACT OF SHIFTING SECONDARY FROM PRIMARY WINDING

\begin{tabular}{|c|c|c|c|c|}
\hline $\begin{array}{c}\text { Angle of } \\
\text { shift }\end{array}$ & $\begin{array}{c}\text { Frequency } \\
f_{\mathrm{r}}, \mathrm{kHz}\end{array}$ & $\begin{array}{c}\text { Injected } \\
P_{\text {core }}, \mathrm{W}\end{array}$ & $\begin{array}{c}\text { Equivalent to } \\
\text { operating } B_{\mathrm{m}}, \mathrm{T}\end{array}$ & $\begin{array}{c}\text { Extra leakage } \\
l_{1}, \mu \mathrm{H}\end{array}$ \\
\hline 0 degree & 12.1 & 0.076 & 0.0096 & 0.0 \\
\hline $30 \mathrm{deg}$. & 11.85 & 60.7 & 0.545 & 1.2 \\
\hline $90 \mathrm{deg}$. & 11.60 & 106.9 & 0.784 & 2.4 \\
\hline $180 \mathrm{deg}$. & 11.50 & 115.0 & 0.826 & 2.9 \\
\hline
\end{tabular}

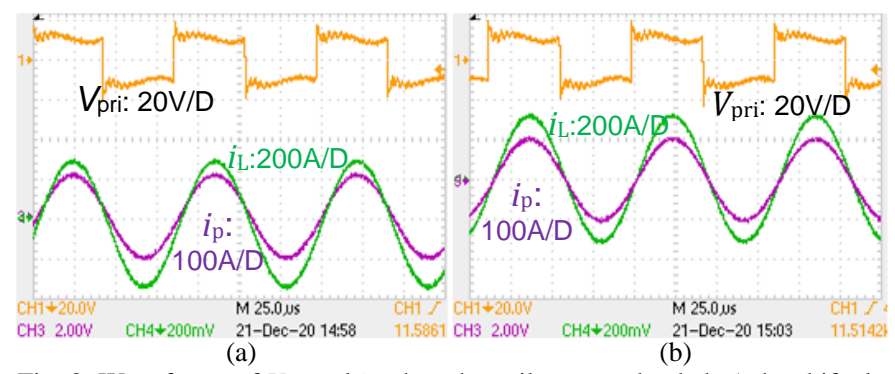

Fig. 8: Waveforms of $V_{\text {pri }}$ and $i_{\mathrm{p}}$ when the coil was not loaded, a) the shifted angle between primary and secondary was $90^{\circ}$ where average value of $V_{\mathrm{CH}}$ was $13.8 \mathrm{~V}$ and $f_{\mathrm{r}}$ was $11.6 \mathrm{kHz}, P_{\text {core }}$ injected was $106.9 \mathrm{~W}$, and, b) for $180^{\circ}$ shift, $V_{\mathrm{CH}}$ was $14.0 \mathrm{~V}, f_{\mathrm{r}}$ was $11.5 \mathrm{kHz}$ and $P_{\text {core }}$ injected was $115.0 \mathrm{~W}$.

Table II details the implication of shifting the secondary winding relative to the primary. Corresponding waveforms of $V_{\text {pri }}, i_{\mathrm{p}}$ and $i_{\mathrm{L}}$ are shown in Fig. 6a and 6b, and in Fig. 8a and 8b. When the secondary was shifted by $180^{\circ}$ with respect to primary (see Fig. 7), it resulted maximum nonuniformity of distribution of flux density. It injected more loss $(24.8 \mathrm{~W} / \mathrm{kg})$ in core and the value of $l_{1}$ was maximum. In actual application (see Fig. 4a), considering sinusoidal excitation, when IHT delivered full power, the calculated value of $P_{\text {core }}$ was $51.6 \mathrm{~W}$. But, because square wave voltage was applied at primary, the core loss would be more [5]. In this set up, at rated current, it was practically found that by adjusting the shifting angle close to 30 degree the requisite loss (here it was $60.7 \mathrm{~W}$ ) could be injected in core. The corresponding waveforms are shown in Fig. 6b. However, the extra loss occurred was centered around the windings in nonuniform manner, a bit localized. Therefore, the cores under consideration should have large thermal conductivity where nanocrystalline material was suitable. The extra loss of $60.7 \mathrm{~W}$ would get reflected in an increase in reading of $V_{\text {pri }}$ i.e., $\Delta V_{P r i}$. At $i_{\mathrm{p}}$ of $75 \mathrm{~A}$, to inject $60.7 \mathrm{~W}$ in core, the value of $\Delta V_{P r i}$ is,

$\Delta V_{\text {pri }}=\frac{60.7}{0.9 i_{p}}=0.89$

This condition was practically established (see Fig. 6b).

Now, the energized inverter was tested for 90 minutes. The enclosure (see Fig. 3) was forced air-cooled. The temperature reading was taken at multiple locations on each core segment as well as on copper windings. At injected core loss density of $13.1 \mathrm{~W} / \mathrm{kg}$ and current density of $3.8 \mathrm{~A} / \mathrm{mm}^{2}$ the maximum temperature noted on core was $81.3{ }^{\circ} \mathrm{C}$ and on windings it was $79.5^{\circ} \mathrm{C}$. The ambient temperature was $33{ }^{\circ} \mathrm{C}$.

\section{FULl LOAD TEST OF IHT WITH SECONDARY SHORTED}

In practical demonstrations detailed above the power drawn from L1 was zero, but IHT fed requisite power to keep the tank circuit in energized state. It could make adverse impact because increase in coil temperature would result erroneous reading.

The error discussed above would be avoided in the proposed method where the complete tank circuit of Fig. 1 is eliminated and the secondary of IHT is shorted. The leakage inductance $\left(L_{\text {Leak }}+l_{1}\right)$ of IHT is now the inductance in the new tank circuit. Here, thermal performance at full load of IHT would be emulated even though the power drawn from it is exactly zero. Using suitable low power SRI, if following conditions are met then requisite core loss would be injected into the core and full load copper loss would remain unchanged:

1. Current $i_{\mathrm{L}}$ and $i_{\mathrm{p}}$ should be same as used for Fig. 6

2. The frequency of current must remain unchanged, and,

3. The angle of $30^{\circ}$ between the windings remains same.

To emulate the full load testing of high power IHT, at its zero output power, one $200 \mathrm{~W}$ low-voltage high-current ZVZCS half-bridge SRI (see Fig. 9) was developed. Transformer TR was used as current multiplier so that low current Mosfets (Q6Q8) could be used. To ensure zero output from IHT, its secondary terminals were shorted. Along with new tank capacitor Cr1, IHT was used as inductance to complete the tank circuit (see Fig. 9). The primary and secondary currents of IHT were kept unchanged as used for results with waveforms shown in Fig. 6 and Fig. 8. The complete low power experimental set up is shown in Fig. 10. The IHT shown here is the same one already used to generate results for Fig. 6b. To inject requisite loss in IHT, $f_{\mathrm{rl}}$ of (18) is Kept unchanged. Capacitor $C_{\mathrm{r}}$ has been accordingly modified to $C_{\mathrm{r} 1}$ (see Table III), so that,

$f_{\text {new }}=f_{\mathrm{r} 1}=\frac{1}{2 \pi \sqrt{\left(L_{\text {leak }}+l_{1}\right) C_{r 1}}}$

The parametric details of the new low power inverter are listed in Table III. The power input to input of TR (see Fig. 9) is,

$P_{\mathrm{TR}} \approx 0.45 V_{\mathrm{CH}} i_{r}$

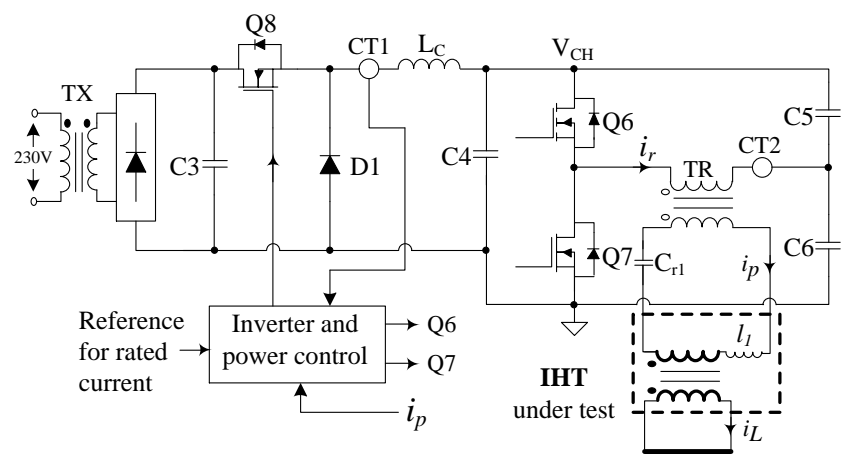

Fig. 9: Schematic of low power half-bridge ZVZCS SRI to emulate as full load testing infrastructure for high power IHT 
Fig. 11 shows two sets of waveforms. Waveforms in Fig. 11a belong to the condition when the two windings were in perfect overlaid condition (see Fig. 5). When the secondary was shifted by 30 degree relative to the primary winding, the corresponding waveforms are shown in Fig. 11b. At desired frequency with respective rated current in both the windings, the shifted shortcircuited secondary would inject around $60.7 \mathrm{~W}$ into the core. Moreover, with rated winding currents the copper loss would also be at its rated value of $94.3 \mathrm{~W}$. Therefore, though, the power drawn from IHT was zero, rated values of $P_{\mathrm{cu}}$ and $P_{\text {core }}$ were ensured in the IHT.

TABLE III

SYSTEM PARAMETERS TO EMULATE FULL LOAD TESTING OF IHT

\begin{tabular}{cc}
\hline \hline Parameter & Value \\
\hline Primary and secondary turns of TR & 8 and 1 \\
Rectified input supply DC voltage $\mathrm{V}_{\mathrm{S}}, \mathrm{V}$ & 55.0 \\
Capacitors C3, $\mu \mathrm{F}$, Voltage, $\mathrm{V}$ & 6000,63 \\
Core material of TR & Ferrite \\
IGBT Q6, Q7, Q8 & IRFP4321 \\
Diode D1 & DSSK 2 X 015A \\
Value of $L_{\text {leak }}$ and stray inductance together, $\mu \mathrm{H}$ & 5.8 \\
Capacitors C4, C5, C6, $\mu \mathrm{F}$ & 100 \\
Tank capacitor Cr1, $\mu \mathrm{F}$ & 30 \\
Primary and secondary current of IHT, A & 75 and 225 \\
Current density in winding conductors, A/mm2 & 3.8 \\
Ambient temperature, ${ }^{0} \mathrm{C}$ & 33.0 \\
\hline \hline
\end{tabular}

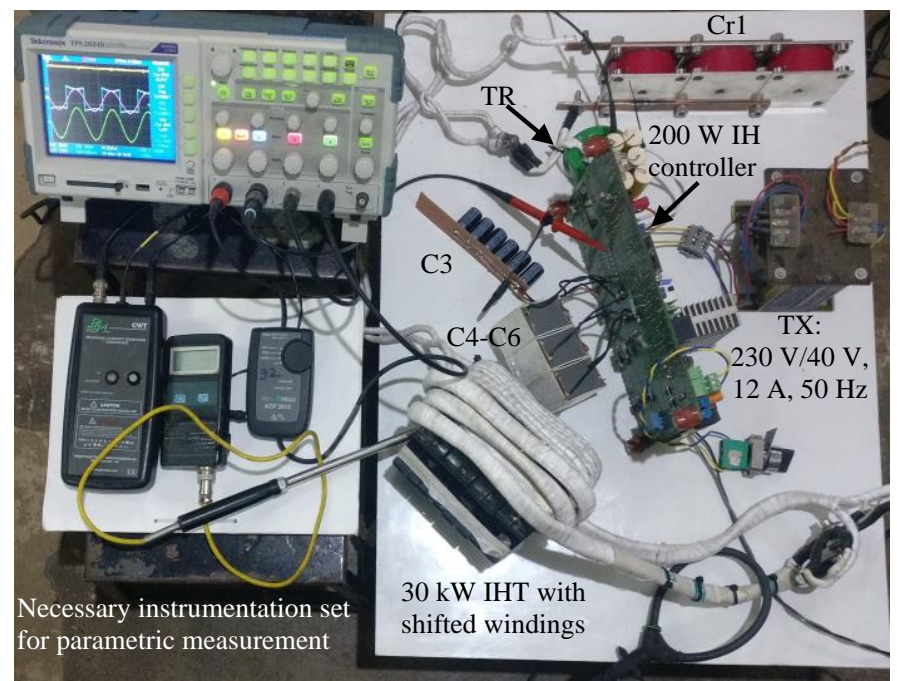

Fig. 10: Complete experimental set up with necessary instrumentation of $200 \mathrm{~W}$ ZVZCS half-bridge SRI to emulate full load testing facility for $30 \mathrm{~kW}$ IHT

The inverter was load tested for 90 minutes. As shown in Fig. 10 , the IHT was kept in open air under natural convection at ambient temperature $33{ }^{\circ} \mathrm{C}$. Using both resistance temperature detector (RTD) and Fluke make (Mini 59) infra-red thermometer, the temperature at different segments of ribbons of nano-crystalline cores and windings were noted. When RTD probes were used, to avoid high frequency effects the temperatures were recorded off line. The readings of both the thermometers were found to be matching with each other. At high core loss density, the maximum temperature on ribbon of core close to primary winding was recorded high at $90.5^{\circ} \mathrm{C}$ and that of copper winding was $85.5{ }^{\circ} \mathrm{C}$. Due to large thermal conductivity of nanocrystalline core material, the temperature variation at different parts of core was within $4{ }^{\circ} \mathrm{C}$.

However, when 4-inch (90 CFM) fan was added to improve air circulation the temperature of core was much lower value at $81{ }^{\circ} \mathrm{C}$ and that of winding was $75^{\circ} \mathrm{C}$. The temperature rise was less than normally considered value of $50^{\circ} \mathrm{C}$.

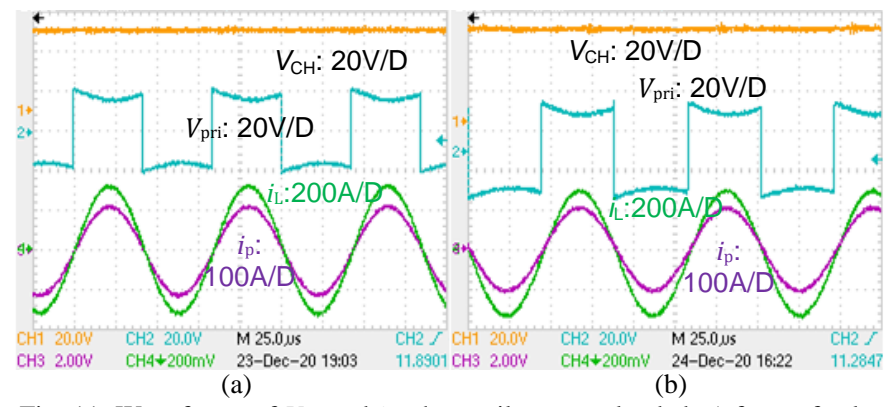

Fig. 11: Waveforms of $V_{\text {pri }}$ and $i_{\mathrm{p}}$ when coil was not loaded, a) for perfectly overlaid windings, the value of $V_{\mathrm{CH}}$ was $35 \mathrm{~V}$ and frequency $f_{r}$ was $11.9 \mathrm{kHz}$, and, b) when secondary was shifted by nearly $30^{\circ}$ where average value of $V_{\mathrm{CH}}$ was $48.1 \mathrm{~V}$ and $f_{r}$ was $11.6 \mathrm{kHz}$. The injected core loss was $56.8 \mathrm{~W}$.

\section{CONCLUSION}

The article analyzed that due to application specific complex load profile, organizing a facility for prolonged testing of large capacity IHT at full load was complex. It further detailed that, even in no load condition, copper loss at primary and secondary was at their respective rated value. The article, after exploring origin of different type of eddy current losses in soft magnetic material, proposed a novel idea of injecting magnetic loss equivalent to the rated core loss value of IHT. It was achieved by mechanically shifting the axis of one winding relative to the other. Initially, the proposed idea was validated, under no load condition where the power delivered by IHT was the copper loss of coil head. The article, with the help of a newly designed low-power half bridge SRI, further strengthened the idea of repeating the test when the secondary of IHT was shorted. The proposed idea needed just $200 \mathrm{~W}$ resonant inverter to emulate the full load testing $30 \mathrm{~kW}$ IHT. However, as it involved nonuniform eddy current loss inside the core, the proposed concept would be more suitable for soft magnetic materials having large thermal conductivity, e.g., nanocrystalline core material.

\section{REFERENCES}

[1] W. G. Hurley, W. H. Wolfe and J. G. Breslin, "Optimized transformer design: Inclusive of high-frequency effects," IEEE Trans. Power Electr., vol. 13, no. 4, pp. 651-659, July 1998.

[2] M. A. Bahmani, T. Thirigner and M. Khareji, "Design methodology and optimization of a medium-frequency transformer for high-power DC-DC applications," IEEE Trans. Ind. Appln., vol. 52, no. 5, pp. 4225-4233, Sept/Oct 2016.

[3] A. K. Paul, "ZVZCS SRI guides optimal use of copper and core for aircooled nanocrystalline transformer for induction heating," IEEE Trans. Ind. Appln., vol. 56, no. 2, pp. 970-978, Mar/Apr 2020.

[4] R. Petkov, "Optimum design of a high-power high-frequency transformer," IEEE Trans. Power Electr., vol. 11, no. 11, pp. 33-42, Jan 1996.

[5] W. Shen, F. Wang, D. Boroyevich and C. Wesley, "High-density nanocrystalline core transformer for high-frequency resonant converter," IEEE Trans. Ind. Appln., vol. 44, no. 1, pp. 213-222, Jan./Feb. 2008.

[6] I. Villar, A. Garcia-Bediaga, U. Viscarret, I. Etxeberria-Otadui and A. Rufer, "Proposal and validation of medium frequency power transformer design methodology," in Proc. IEEE ECCE 2011, pp. 3792-3799, IEEE. 
[7] A. K. Paul, "Structured protection measures for better use of nanocrystalline cores in air-cooled medium frequency transformer for induction heating," IEEE Trans. Ind. Electron., vol. 58, issue 5, pp. 3898-3905, May 2021.

[8] M. Mogorovic and D. Dujic, "100 kW, $10 \mathrm{kHz}$ medium-frequency transformer design optimization and experimental verification," IEEE Trans. Power Electron., vol. 34, issue 2, pp.1696-1708, Feb 2018.

[9] W. G. Odendaal and J. A. Ferreira, "A Thermal model for high-frequency magnetic components," IEEE Trans. Ind. Appln., vol. 35, no. 4, pp. 924931, Jul./Aug. 2016.

[10] Z. Ouyang, J. Zhang and W. G. Hurley, "Calculation of leakage inductance of high-frequency transformers," IEEE Trans. Power Electron., vol. 30, no. 10, pp. 5769-5775, Oct 2015.

[11] L. Shih, Y. Liu, and H. Chiu, "A novel hybrid mode control for a phaseshift full-bridge converter featuring high efficiency over a full-load range," IEEE Trans. Power Electron., vol. 34, no. 3, pp. 2794-2804, Mar 2019.

[12] J. -M. Choi, B. -J. Byen, Y. -J. Lee, D. -H. Han, H. -S. Kho and G. -H. Choe, "Design of leakage inductance in resonant DC-DC converter for electric vehicle charger," IEEE Trans. Magnetics, vol. 48, no. 11, pp. 4417-4420, Oct 2012.

[13] O. Lucia, P. Maussion, E. J. Dede and J. M. Burdio, "Induction heating technology and its applications: Past developments, current technology, and future trends," IEEE Trans. Ind. Electron., vol. 61, no. 5, pp. $2509-$ 2520, May 2014.

[14] A. K. Paul, "Active-controlled passive distribution of power offers efficient heat treating solution for quality arc welding joints of steel pipes," IEEE Trans. Ind. Appln., vol. 55, no. 5, pp. 4958-4966, Sep./Oct, 2018.

[15] Advanced Technology and Materials Co., "Datasheet of core type 1G1207030, part no. C.NA004.1200403-P062".

[16] Y. Zhang, M. C. Cheng and P. Pillay, "Magnetic characteristics and excess eddy current losses," in IEEE IAS Conf. Rec., pp. 1-5, 2009.

[17] G. Bertotti, "General properties of power losses in soft magnetic materials," IEEE Trans. Mag., vol. 24, no. 1, pp. 621-630, Jan 1988.

[18] Z. Li, K. Yao, D. Li, X. Ni and Z. Lu, "Core loss analysis of Finemet type nanocrystalline alloy ribbon with different thickness," Progress in Natural Science: Materials Intl., vol. 27, no. 5, pp. 588-592, 2017.

[19] M. Szewczyk, K. Kutorasiński and W. Piasecki, "Quantitative analysis of high-frequency material properties in thin-ribbon magnetic cores," IEEE Trans. Magnetics, vol. 51, no. 8, pp. 1-6, Aug. 2015.

[20] T. Kauder and K. Hameyer, "Performance factor comparison of nanocrystalline, amorphous and crystalline soft-magnetic materials for medium frequency applications," IEEE Trans. Magnetics, vol. 53, no. 11, pp. 1-4, Nov 2017.

[21] F. Flohrer and G. Herzer, "Magnetization loss of nanocrystalline soft magnets," in Intl. Conf. on Rapidly Quenched and Metastable Materials, Conf. Series 144(2009) 012075, IOP Publishing, pp. 1-5, 2009.

[22] M. S. Sanjarinia, S. Saadatmand, P. Shamsi and M. Ferdowsi, "Analysis of various transformer structures for high frequency isolation applications," in North American Power Symp., pp. 1-6, IEEE, 2019. 